Original paper

\title{
Radiobiological quantities in proton-therapy: Estimation and validation using Geant4-based Monte Carlo simulations
}

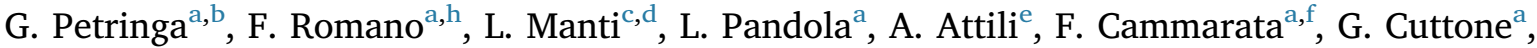 \\ G. Forte ${ }^{\mathrm{a}, \mathrm{f}}$, L. Manganaro ${ }^{\mathrm{e}}$, J. Pipek ${ }^{\mathrm{g}}$, P. Pisciotta ${ }^{\mathrm{a}, \mathrm{b}}$, G. Russo ${ }^{\mathrm{a}, \mathrm{f}}$, G.A.P. Cirrone ${ }^{\mathrm{a}, \mathrm{g}, *}$ \\ ${ }^{a}$ INFN-LNS. Istituto Nazionale di Fisica Nucleare - Laboratori Nazionali del Sud, Via S. Sofia 62, 95123 Catania, Italy \\ ${ }^{\mathrm{b}}$ Dipartimento di Fisica e Astronomia, Universitá degli Studi di Catania, Via S. Sofia 64, 95123 Catania, Italy \\ ${ }^{\mathrm{c}}$ Dipartimento di Fisica E. Pancini, Universitá degli Studi Federico II di Napoli, Via Cinthia, I-80126 Napoli, Italy \\ ${ }^{\mathrm{d}}$ INFN-NA, Istituto Nazionale di Fisica Nucleare, Sezione di Napoli, Complesso Universitario di M. S. Angelo, Via Cintia, I-80126 Napoli, Italy \\ ${ }^{\mathrm{e}}$ INFN-TO, Istituto Nazionale di Fisica Nucleare, Sezione di Torino, Torino, Italy \\ ${ }^{\mathrm{f}}$ IBFM-CNR, Institute of Molecular Bioimaging and Physiology - National Research Council, Cefalù, PA, Italy \\ ${ }^{\mathrm{g}}$ ELI-Beamline Project, Inst. Physics, ASCR, PALS Center, Prague, Czech Republic

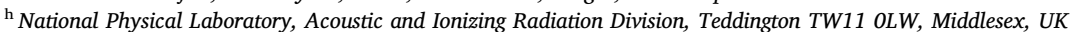

\section{A R T I C L E I N F O}

\section{Keywords:}

Monte Carlo

Proton

Radiotherapy

Geant4

Radiobiological model

RBE

Survival fraction

\begin{abstract}
A B S T R A C T
Purpose: The Geant4 Monte Carlo simulation toolkit was used to reproduce radiobiological parameters measured by irradiating three different cancerous cell lines with monochromatic and clinical proton beams.

Methods: The experimental set-up adopted for irradiations was fully simulated with a dedicated open-source Geant4 application. Cells survival fractions was calculated coupling the Geant4 simulations with two analytical radiobiological models: one based on the LEM (Local Effect Model) approach and the other on a semi-empirical parameterisation. Results was evaluated and compared with experimental data.

Results and conclusions: The results demonstrated the Geant4 ability to reproduce radiobiological quantities for different cell lines.
\end{abstract}

\section{Introduction}

An increasing number of radiotherapy facilities make, use of accelerated charged beams to treat tumours. Since 1954, approximately 174,000 patients were treated with ions: the vast majority with protons, but heavier particles such as ${ }^{12} \mathrm{C}$ ions have also been used [1]. The main reason for this rapid growth lies in the better physical selectivity offered by protons compared to electrons and gamma rays. In addition to the advantages offered by the peculiar depth-dose profile, protons also show an enhanced biological effectiveness in cell killing [2] towards the end of the clinical SOBP (Spread Out Bragg Peak). This prompted research works in assessing the radioresponse of known radioresistant cancer cell lines such as those from gliomas [3]. In view of such advantageous dose profiles, protons are also being considered for treatment of breast cancer where their use would limit radiation-induced cardiovascular disease, which is known to be a late-occurring noncancer effect in radiotherapy [4]. In addition, radiation is known to alter the metastatic potential of cancer cells by modifying the infiltrating phenotype in the tumour microenvironment [5]. In this regard, it is interesting to study the radioresponse of a known metastatic breast cancer cell line such as MDA-MB-231. The biological effect of ionising radiation is quantified by means of the Relative Biological Effectiveness (RBE). RBE is defined as the ratio of the absorbed dose of a reference radiation, typically $250 \mathrm{kVp}$ x-rays or ${ }^{60} \mathrm{Co}$ gamma rays [6], which produces a given biological effect, to the absorbed dose of the test radiation producing the same effect. The clinical application of RBE is not straightforward as its value is a function of particle type, energy, dose, dose per fraction, cell or tissue type [6]. In the last years several attempts were done to develop models to evaluate the RBE in clinical cases. Among others, the Local Effect Model (LEM) [7] and the Microdosimetric Kinetic Model (MKM) [8] are the computational radiobiological approaches most widely used in the clinical radiotherapy with carbon beams. The LEM model was fully integrated in the analytical treatment planning system TRiP98 (TReatment planning for Particles, 1998) and successfully tested for clinical application at the CNAO (Pavia, I) [9], HIT (Heidelberg, D) and Medaustron (Wien Neustadt, A) [10] hadrontherapy facilities for the optimization of the biological dose of carbon ions beams $[11,12]$.

\footnotetext{
* Corresponding author.

E-mail address: pablo.cirrone@lns.infn.it (G.A.P. Cirrone).
} 
Monte Carlo (MC) simulations are able to simulate hadronic elastic and inelastic processes when particles penetrate matter and the transport of secondaries produced in such interactions. Many MC codes are available today but only a few have the capability to evaluate proton RBE distributions [13-15]. In a recent publication, Mairani et al. [16] discussed an approach to calculate the RBE-weighted dose in case of two opposed and superimposed ${ }^{12} \mathrm{C}$ ion beams.

This paper demonstrate the potentialities of Geant 4 in the calculation of the in vitro Survival Fraction (SF) and of the RBE in therapeutic proton beams and specifically in a clinical proton beam used for eye protontherapy.

Two different approaches were developed to estimate the RBE: a computational method, which couples Geant4 with the LEM model (version III) [17], and a parametrized approach of the LQM, inspired by the McNamara work [21] and implemented for proton beam irradiations. Both approaches were applied to reproduce survival curves measured in three experiments conducted at the CATANA (Centro di Adroterapia ed Applicazioni Nucleari Avanzate) protontherapy facility of INFN-LNS (Catania, I) [22].

The first campaign, carried out by Chaudhary et al. [3], consisted in the irradiation of U87 radioresistant human glioma cells at six different depths along a monochromatic $(62 \mathrm{MeV})$ and a modulated clinical proton Spread Out Bragg Peak (SOBP) beam. A new series of experiments were then performed, in which the more radiosensitive prostate cancer cell line DU145 were irradiated together with the breast cancer cell line MDA-MB-23 along the SOBP. The whole experimental set-up of the three campaigns, including the diagnostic elements of the transport beam line and the cell irradiation conditions, were accurately simulated using the public release of the Geant4 Hadrontherapy advanced example $[23,24]$.

\section{Simulations}

\subsection{Simulated set-up}

Hadrontherapy is a Geant4 -based application, specifically developed for dosimetric and radiobiological studies with protons and ions beams. It is able to fully simulate the CATANA eye protontherapy beamline with all its beam transport elements: scattering and modulation systems for spatial and energy distribution beam definition, collimators and detectors for the online beam monitoring. At the end of the beam line, a voxelized detector reproduces a typical water tank for dose curves reconstruction. In order to reproduce the adopted experimental condition, the phantom was divided in slices of dimension $400 \times 400 \times 0.01 \mathrm{~mm}^{3}$. Dose, fluence, dose-averaged LET (Linear Energy Transfer), RBE and all quantities necessaries for their estimation were scored during the simulation in each slice. The primary proton beam was simulated with a Gaussian energy distribution, centered at the nominal energy of $62.45 \mathrm{MeV}$ and a standard deviation of $0.25 \mathrm{MeV}$. The beam spot was assumed circular and modelled as a bivariate Gaussian distribution having standard deviations of $5 \mathrm{~mm}$. Beam angular distribution was assumed with a standard deviation of 0.028 degree. A total of $10^{7}$ histories were simulated in each run. The production cut for secondary protons, gamma and electrons was fixed at $0.01 \mathrm{~mm}$. All calculations were carried out using the 10.03.p02 version of Geant4 [25]. Fig. 1 shows the simulated SOBP in water and the corresponding primary proton dose-averaged LET. The corresponding depth dose, acquired in water with a Markus Advanced ionisation chamber, is also displayed.

\subsection{Survival fraction curves calculated coupling the Local Effect Model (LEM) to Geant4}

A method was developed to assess the biological damages produced by proton beams in terms of survival fraction curves, i.e of the number of cells able to survive after the irradiation at different dose. The approach is based on the combined use of Monte Carlo Geant4 simulations (to calculate the doses deposited and the energy spectra of particles interacting with cells) and of the Survival analytical code [28].Survival provides the implementation of different versions of LEM (version I, II and III) and the MKM model. Different numerical evaluation approaches are also implemented in Survival: MC numerical methods and a set of faster analytical approximations. The Survival toolkit is also able to reproduce the RBE as a function of LET for different ions (proton, $\mathrm{He}, \mathrm{C}, \mathrm{O}$ and $\mathrm{Ne}$ ) and different cell lines. Survival calculate ion- and cell-specific look-up tables (LUT). LUTs contain the $\alpha$ and $\beta$ parameters of the survival Linear-Quadratic model (Eq. (3)) as function of the energy deposited (Edep) and ion kinetic energies (Ekin). On the other side, Monte Carlo simulations permit the calculation of the Edep and Ekin distributions that, coupled with the radiobiological responce model, allow the final and calculation of a survival curve. The calculation of the LUTs from the Survival code is described in 2.2.1 Section. The computation of $\alpha$ and $\beta$ is described in 2.2.3.

\subsubsection{Look up tables calculation}

The LEM $[17,26,27]$ is a model used to estimate the biological effects of ions starting from the response of cells or tissues to photons: it hence permits to exploit a huge database already collected in conventional radiotherapy. The LEM uses the so called local-dose concept i.e., for a given set of particle trajectories, the expectation value of the energy deposition at any position in the radiation field. LEM is based on the fundamental hypothesis that equal local doses produce the same effect, irrespective of the radiation quality. Several refinements of the model have been introduced over time, known as LEM II $[18,19]$, LEM III [20] and LEM IV [19]. In the present analysis we adopted a fast implementation of the LEM based on the Low Dose Approximation approach allowing the derivation of the parameters $\alpha$ and $\beta$ described in [20]. Furthermore this approximation can be applied to all versions of the LEM. Specific Look-Up Tables (LUTs), each for a specific cell-line and incident ion (from $\mathrm{Z}=1$ to $\mathrm{Z}=8$ ), have been generated using the version III of the LEM model. Each LUT contains the $\alpha$ and $\beta$ values as a function of ion LETs and kinetic energy values.

\subsubsection{Estimation of survival curves and calculation of then weighted-RBE}

The estimation of the survival curve carried out in this paper was performed evaluating the average $\langle\alpha\rangle$ and $\langle\beta\rangle$ parameters of the irradiation and using the mixed-field formalism derived from the theory of the Dual Radiation Action (TDRA) [29]. The kinetic energy and the LET value of any primary ion and of the secondaries generated in each slice of the simulated water phantom were retrieved at each simulation step. The corresponding values of $\alpha_{i}$ and $\beta_{i}$, for each specific ion $i$ with a kinetic energy $E_{i}$ and a released dose $D_{i}$, were calculated by direct linear interpolation of the LUTs, using $E_{i}$ as the independent variable. All interpolated $\alpha_{i}$ and $\beta_{i}$ were then weighted according to the TDRA to derive the average values $\langle\alpha\rangle$ and $\langle\beta\rangle$ in each slice using the formulas:

$\langle\alpha\rangle=\frac{\sum_{i} \alpha_{i} \cdot D_{i}}{\sum_{i} D_{i}}$

$\langle\beta\rangle=\left(\frac{\sum_{i} \sqrt{\beta_{i}} \cdot D_{i}}{\sum_{i} D_{i}}\right)^{2}$

The flowchart of the implemented calculations is reported in Fig. 2.

This approach, entirely included in the Hadrontherapy advanced example, allowed to take into account, at run time, the complexity due to the presence of a mixed field. The final Survival Fraction curves were then calculated according to the L-Q model:

$S F=e^{-\left(\langle\alpha\rangle D+\langle\beta\rangle D^{2}\right)}$ 


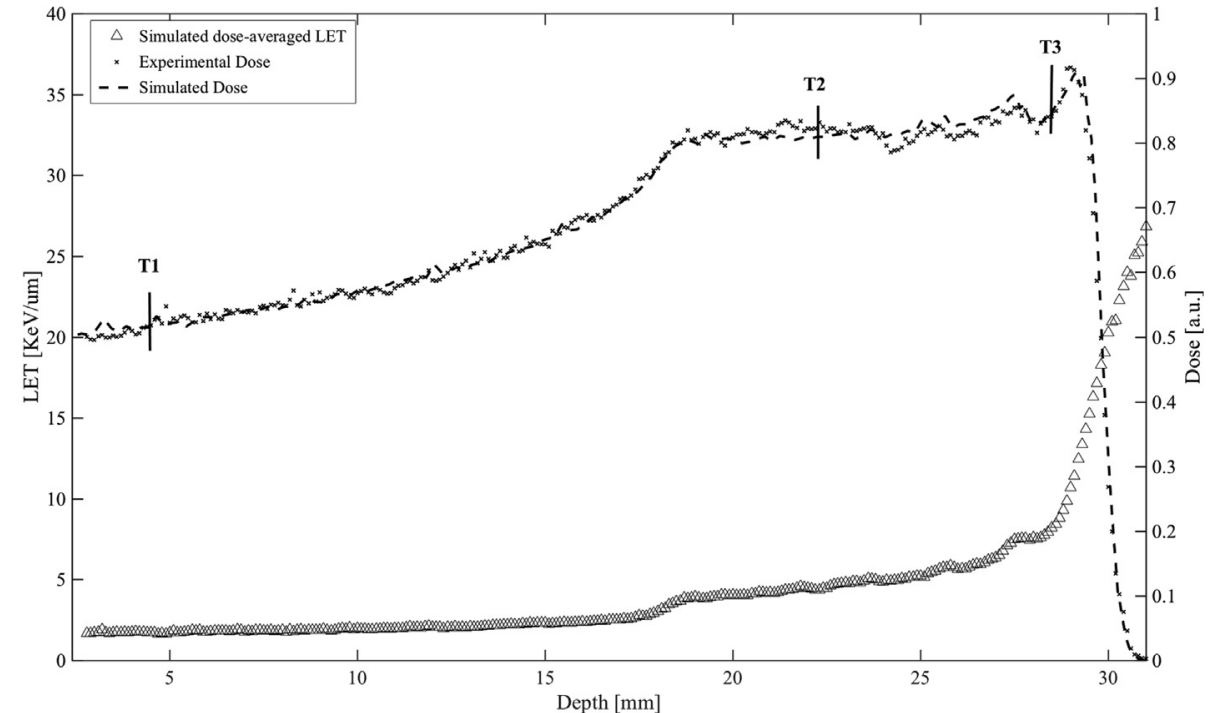

Fig. 1. Proton Spread-Out Bragg Peak (SOBP) used for cells irradiations, measured in water with a PTW (Freiburg, D) Markus advanced ionisation chamber (dotted line) and simulated (dashed line) with the Hadrontherapy Geant4-based application. DU145 cells were placed at points $\mathrm{T} 1$, T2 and T3 corresponding to the depths of 5,21 and $28.9 \mathrm{~mm}$ in water, respectively. Triangles represent the dose averaged LET profiles.
For a give voxel, the following information are retrieved at each particle step:

o Atomic number $Z$

O Atomic mass A

o Kinetic Energy E

o Deposited Energy dE

o Linear Energy Transfer LET

○ Step Length $\mathrm{dx}$
Precompiled LUT (containing the $\alpha$ and $\beta$ values) are recalled step-by-step for each particle.

The $\alpha$ and $\beta$ parameters are then calculated by an interpolation procedure applied at the LUT values and based on particle energy $E$ and LET.
The output variables, calculated at the end of a run are:

$0 \alpha_{\mathrm{D}}$, and $\beta_{\mathrm{D}}$, parameters

o Survival Fraction SF

o Relative Biological EffectivenessRBE

O Physical dose $D_{\text {phys }}$

O Biological dose $\mathrm{D}_{\text {bio }}$

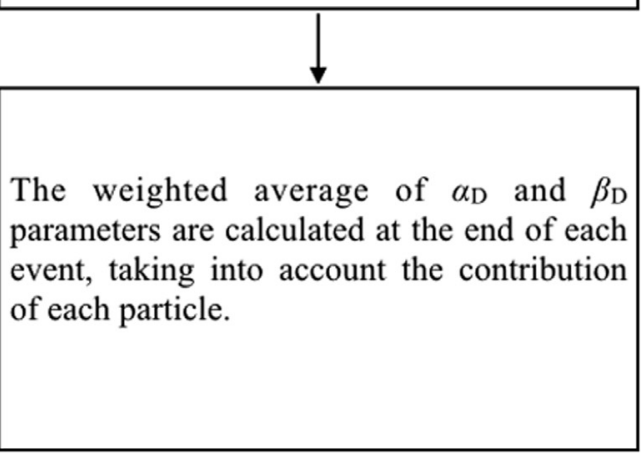

Fig. 2. Outline of the main steps to compute the RBE using the LEM coupled to Geant4 inside Hadrontherapy example.

for a specific released dose D. We remark that the way their coupling of the Monte Carlo algorithm with the LUTs produced by the Survival code allows the use of different radiobiological models, not only the LEM.

\subsection{Implementation of the parameterized $L-Q$ model}

Using an alternative approach, we applied a parameterised model to derive the same survival curve obtained applying LEM (Section 2.2). A clear correlation, in fact, exists between the local energy deposition, as expressed in terms of LET, and the outcome of irradiation at the molecular and cellular level [2]. Dose-averaged LET $\left(\mathrm{LET}_{d}\right)$ represents a reasonable approximation of the physical characteristics of the radiation field for protons up to the depth at which the Bragg peak occurs [30].

In a recent paper, McNamara et al. [21] analyzed an extremely wide set of radiobiological experimental data and derived a phenomenological model able to predict the proton RBE when the released dose $\mathrm{D}_{p}$, the $\mathrm{LET}_{d}$ and the $\alpha / \beta$ ratio for the reference radiation and given cell line are known. The model permits the calculation of the RBE values as:

$R B E=\frac{1}{2} D_{p} \sqrt{R^{2}+4 D_{p} R\left(p_{0}+\frac{p_{1}}{R} \cdot L E T_{D}+4 D_{p}^{2}\left(p_{2}-p_{3} \sqrt{R} \cdot L E T_{D}\right)^{2}\right)}-R$

Here $\mathrm{p}_{0}, \mathrm{p}_{1}, \mathrm{p}_{2}$ and $\mathrm{p}_{3}$ are the fit parameters, $\mathrm{R}$ is the $\left(\frac{\alpha}{\beta}\right)_{X}$ ratio while $\mathrm{LET}_{D}$ can be calculated using Monte Carlo and applying the following formula for each slice of the water phantom [31]:

$L_{E T}=\frac{\sum_{i} L_{i} \varepsilon_{i}}{\sum_{i} \varepsilon_{i}}$

In Eq. (5), $\mathrm{L}_{i}$ is the mean energy loss per step length of the primary and/or secondary ions at the step $\mathrm{i}$ and $\varepsilon_{i}$ is the corresponding energy deposited. The fit parameters of Eq. (4) have been estimated to be $\mathrm{p}_{0}=0.99, \mathrm{p}_{1}=0.39, \mathrm{p}_{2}=1.10$ and $\mathrm{p}_{3}=0.0038[21]$. 

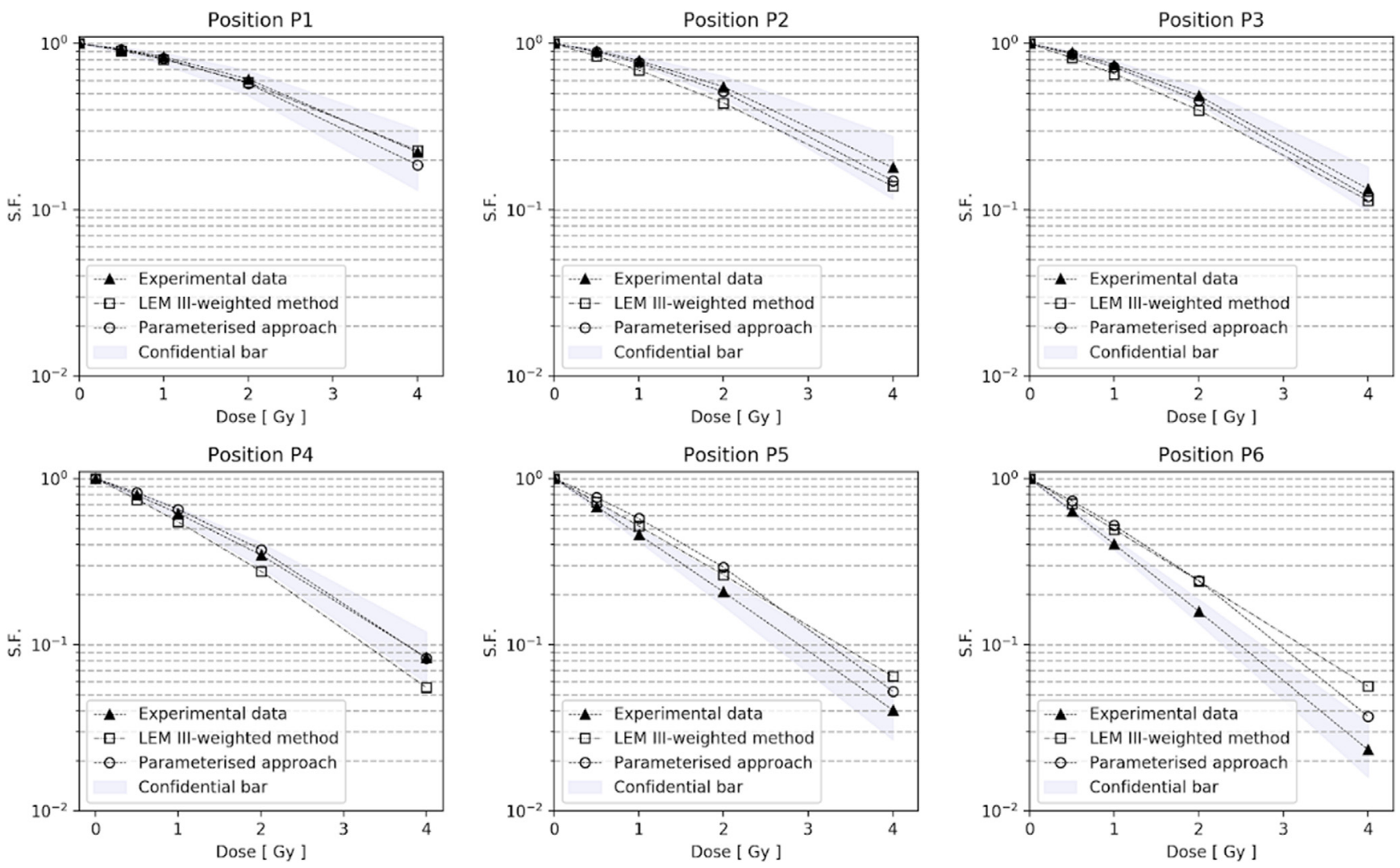

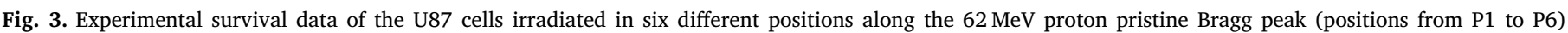

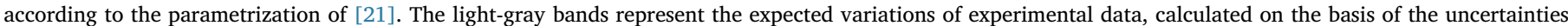
reported $\alpha$ and $\beta$. Survival curves calculated using the LEM (open squares) and parameterized approach (open circles), are also reported.
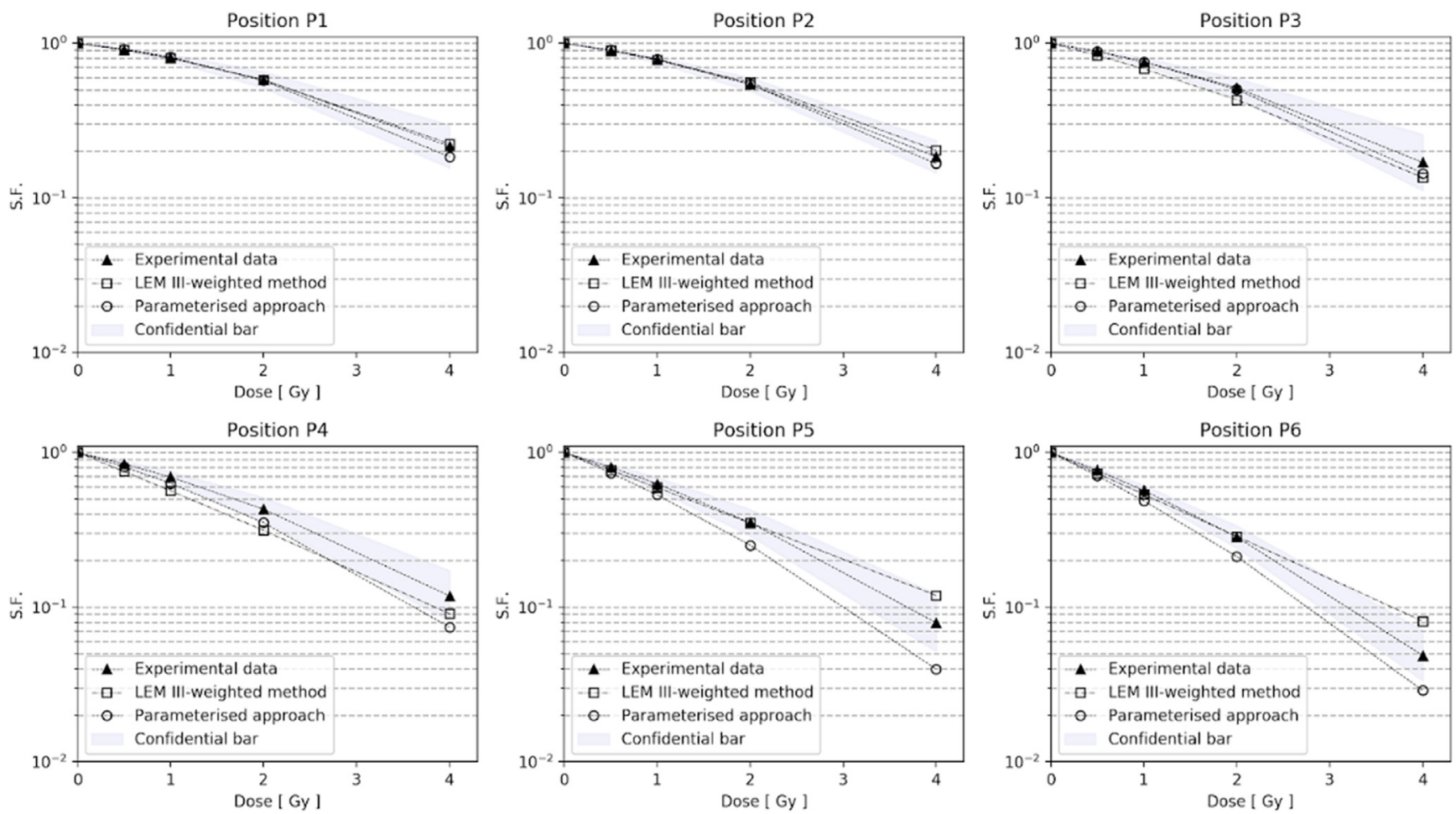

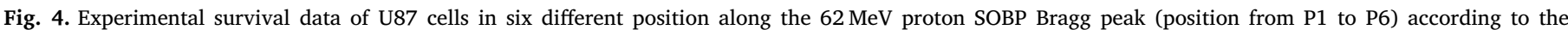

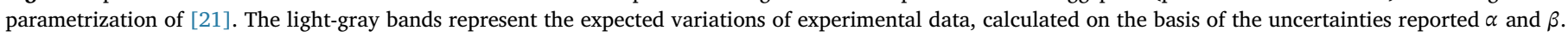
Survival curves calculated using the LEM (open squares) and parameterized approach (open circles), are also reported. 
Table 1

Alpha and Beta parameters calculated using the two approaches: LEM III weighted method and parametrized; Experimental data are also reported.

\begin{tabular}{|c|c|c|c|c|c|c|c|}
\hline \multicolumn{8}{|c|}{ U87 - Pristine } \\
\hline & & Position P1 & Position P2 & Position P3 & Position P4 & Position P5 & Position P6 \\
\hline \multirow[t]{2}{*}{ Experimental results } & alpha $\left[G y^{-1}\right]$ & $0.12 \pm 0.04$ & $0.17 \pm 0.04$ & $0.22 \pm 0.04$ & $0.44 \pm 0.08$ & $0.77 \pm 0.09$ & $0.90 \pm 0.07$ \\
\hline & beta $\left[G y^{-2}\right]$ & $0,064 \pm 0.009$ & $0.065 \pm 0.017$ & $0.071 \pm 0.009$ & $0.045 \pm 0.002$ & $0.008 \pm 0.003$ & $0.010 \pm 0.006$ \\
\hline \multirow[t]{2}{*}{ Parameterized approach } & alpha $[G \hat{y}-1]$ & $0.1321 \pm 0.0001$ & $0.1900 \pm 0.0002$ & $0.2550 \pm 0.0003$ & $0.3612 \pm 0.0004$ & $0.4950 \pm 0.0005$ & $0.5911 \pm 0.0006$ \\
\hline & beta $\left[G y^{-2}\right]$ & $0.0751 \pm 0.0002$ & $0.0720 \pm 0.0006$ & $0.0711 \pm 0.0003$ & $0.064 \pm 0.003$ & $0.061 \pm 0.002$ & $0.051 \pm 0.003$ \\
\hline \multirow[t]{2}{*}{ LEM III weighted method } & alpha $\left[G y^{-1}\right]$ & $0.1711 \pm 0.0002$ & $0.3320 \pm 0.0003$ & $0.3811 \pm 0.0004$ & $0.5621 \pm 0.0005$ & $0.651 \pm 0.0006$ & $0.651 \pm 0.0006$ \\
\hline & beta $\left[G y^{-2}\right]$ & $0.0501 \pm 0.0005$ & $0.4121 \pm 0.0005$ & $0.0411 \pm 0.0005$ & $0.0410 \pm 0.0007$ & $0.0090 \pm 0.0009$ & $0.0090 \pm 0.0005$ \\
\hline \multicolumn{8}{|c|}{ U87 - SOBP } \\
\hline & & Position P1 & Position P2 & Position P3 & Position P4 & Position P5 & Position P6 \\
\hline \multirow[t]{2}{*}{ Experimental results } & alpha $\left[G y^{-1}\right]$ & $0.16 \pm 0.04$ & $0.19 \pm 0.04$ & $0.22 \pm 0.04$ & $0-31 \pm 0.08$ & $0.41 \pm 0.09$ & $0.50 \pm 0.07$ \\
\hline & beta $\left[G y^{-2}\right]$ & $0.056 \pm 0.015$ & $0.058 \pm 0.051$ & $0.064 \pm 0.016$ & $0.056 \pm 0.035$ & $0.056 \pm 0.042$ & $0.064 \pm 0.033$ \\
\hline \multirow[t]{2}{*}{ Parameterized approach } & alpha $\left[G y^{-1}\right]$ & $0.131 \pm 0.001$ & $0.160 \pm 0.002$ & $0.211 \pm 0.002$ & $0.392 \pm 0.004$ & $0.572 \pm 0.006$ & $0.570 \pm 0.006$ \\
\hline & beta $\left[G y^{-2}\right]$ & $0.0701 \pm 0.0002$ & $0.071 \pm 0.0006$ & $0.0691 \pm 0.0003$ & $0.063 \pm 0.003$ & $0.058 \pm 0.003$ & $0.058 \pm 0.003$ \\
\hline \multirow[t]{2}{*}{ LEM III weighted method } & alpha $\left[G y^{-1}\right]$ & $0.1711 \pm 0.0002$ & $0.1800 \pm 0.0003$ & $0.3410 \pm 0.0004$ & $0.3410 \pm 0.0004$ & $0.5320 \pm 0.0005$ & $0.6210 \pm 0.0006$ \\
\hline & beta $\left[G y^{-2}\right]$ & $0.0501 \pm 0.0005$ & $0.0510 \pm 0.0004$ & $0.0420 \pm 0.0004$ & $0.0401 \pm 0.0004$ & $0.0030 \pm 0.0001$ & $0.0021 \pm 0.0002$ \\
\hline
\end{tabular}

Table 2

Alpha and Beta parameters calculated using LEM III and parametrized approaches; Experimental data are also reported.

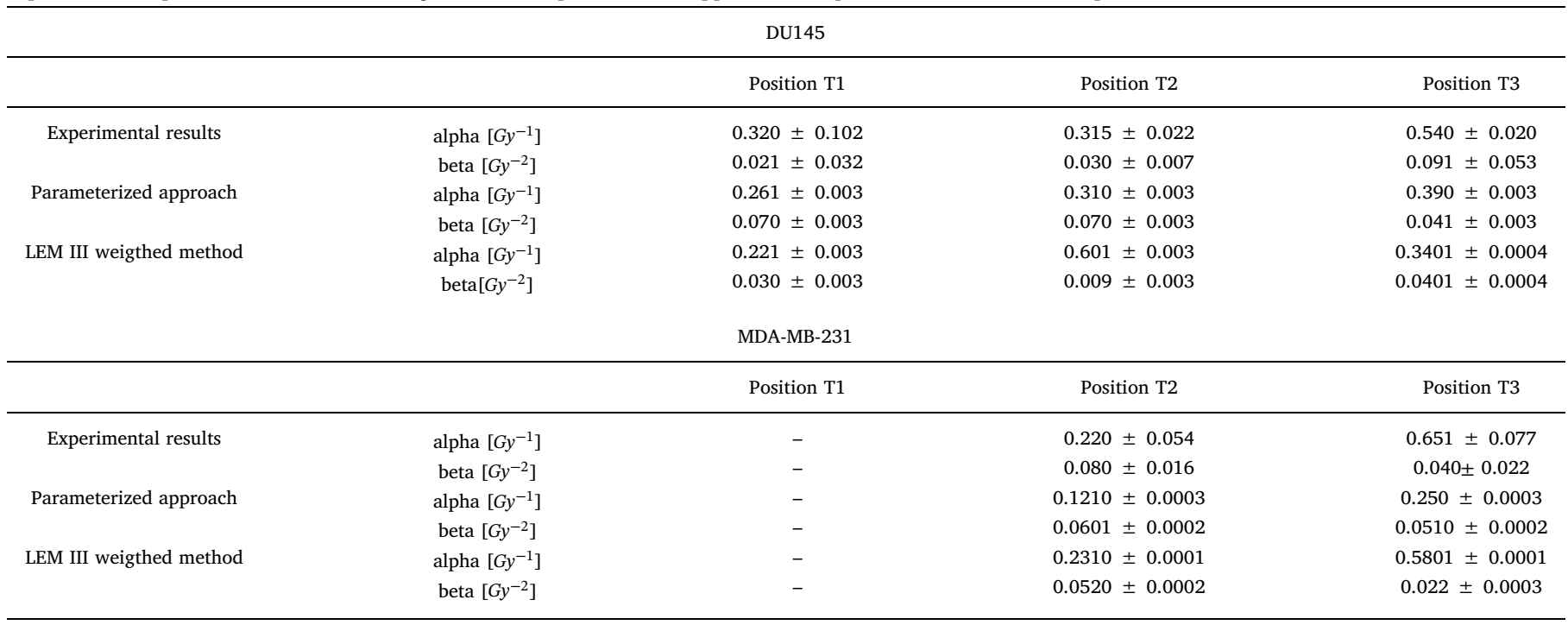

\section{Irradiation and experimental set-up}

We experimentally investigated the response to proton irradiation of radioresistant human glioma (U87), of the relatively more radiosensitive prostate cancer DU145 and of breast cancer cell line MDA-MB231. U87 were irradiated in an experimental campaign conducted at the CATANA protontherapy facility of INFN-LNS. Irradiations were carried out in six different positions (P1, P2, P3, P4, P5 and P6) along a pristine Bragg peak (corresponding to LET values of 1.1, 4.0, 7.0, 11.9, 18.0 , and $22.6 \mathrm{keV} / \mu \mathrm{m}$, respectively) and in six different positions along a modulated spread out Bragg peak (corresponding to LET values of 1.2, 2.6, 4.5, 13.4, 21.7 and $25.9 \mathrm{keV} / \mu \mathrm{m}$.). Details on U87 campaign can be found in Chaudhary et al. [3]. DU145 and MDA-MB-231 were irradiated at the same beam line during two different experimental campaigns. DU145 irradiations were conducted along a clinical proton SOBP in three positions (T1, T2 and T3) corresponding to the depths of 5,21 and $28.9 \mathrm{~mm}$ in water (and LET values of 1.96, 4.24 and $20.62 \mathrm{keV} / \mu \mathrm{m}$ ). The MDA-MB-231 were irradiated in two positions only (T2 and T3), corresponding to mid of a clinical SOBP and the distal part of the same curve. In each position doses of $0.5,1,2$ and $4 \mathrm{~Gy}$ were delivered to both cell lines. The experimental SOBP adopted for irradiation is shown in Fig. 1 together with the simulated one and the corresponding dose-average LET distribution. Absolute dosimetry was performed in water by means of a plane-parallel PTW 34045 advanced Markus ionisation chamber, according to the International Atomic Energy Agency Technical Report Series 398 Code of practice [32]. Dose measurements were carried out at a depth corresponding to the middle of the SOBP, using a reference $25 \mathrm{~mm}$ diameter circular collimator. The clinical proton beam calibration was performed before each irradiation; the variation of beam calibration between the experimental runs resulted to be within 3\%. Details on the irradiation beam lines, dosimetric procedures and related uncertainties for irradiation conditions can be found elsewhere [22].

\section{Results}

Survival curves for U87, DU145 and MDA-MB-231 cell lines were reproduced according to LEM III and the parameterised approaches. Results were compared against the experimental survival curves. 

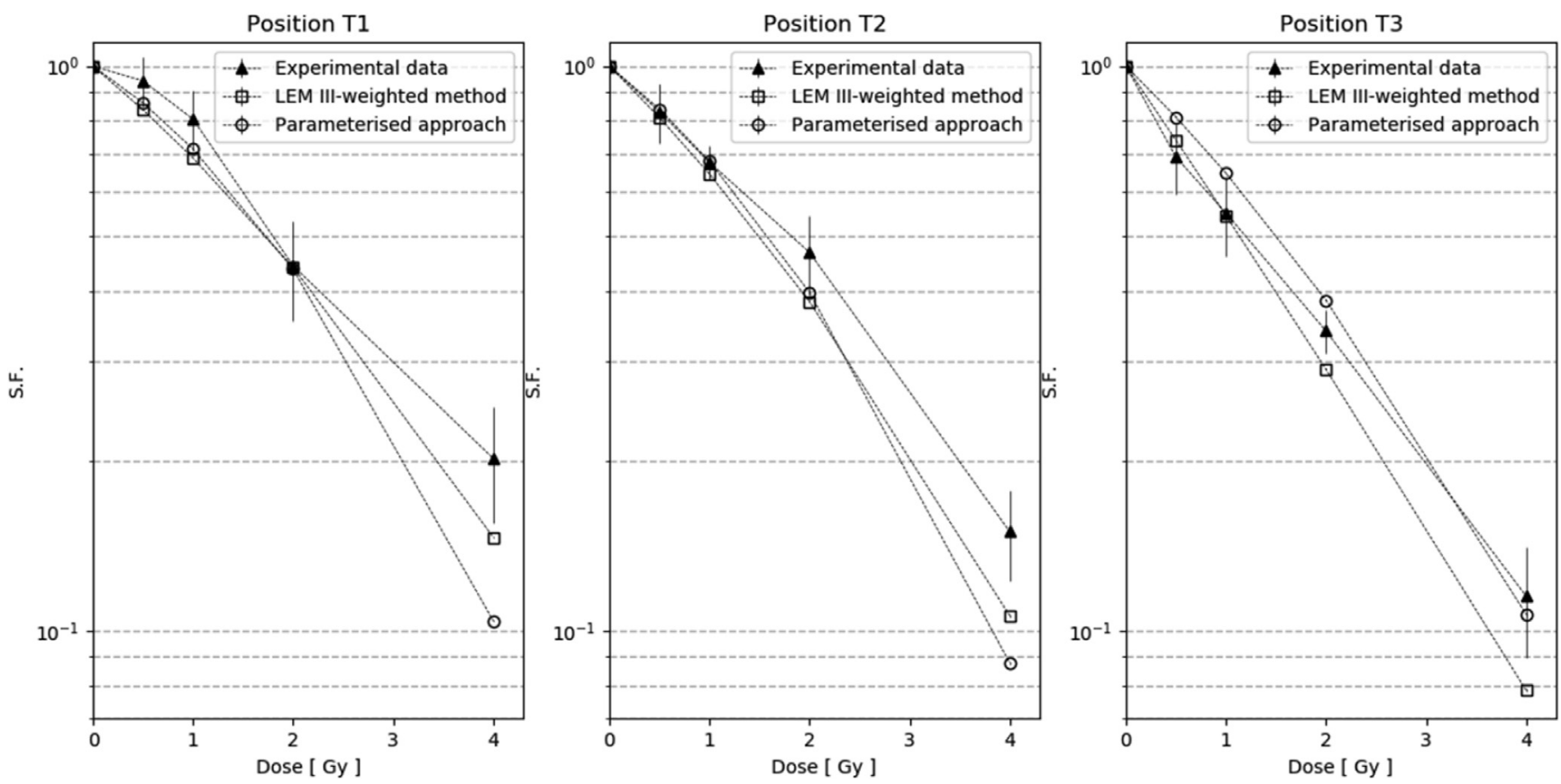

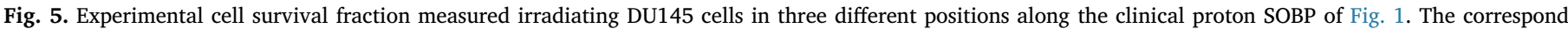
curves calculated by using the Monte Carlo LEM III-weighted method (dashed lines) and the parameterised approach (dotted lines), are reported.
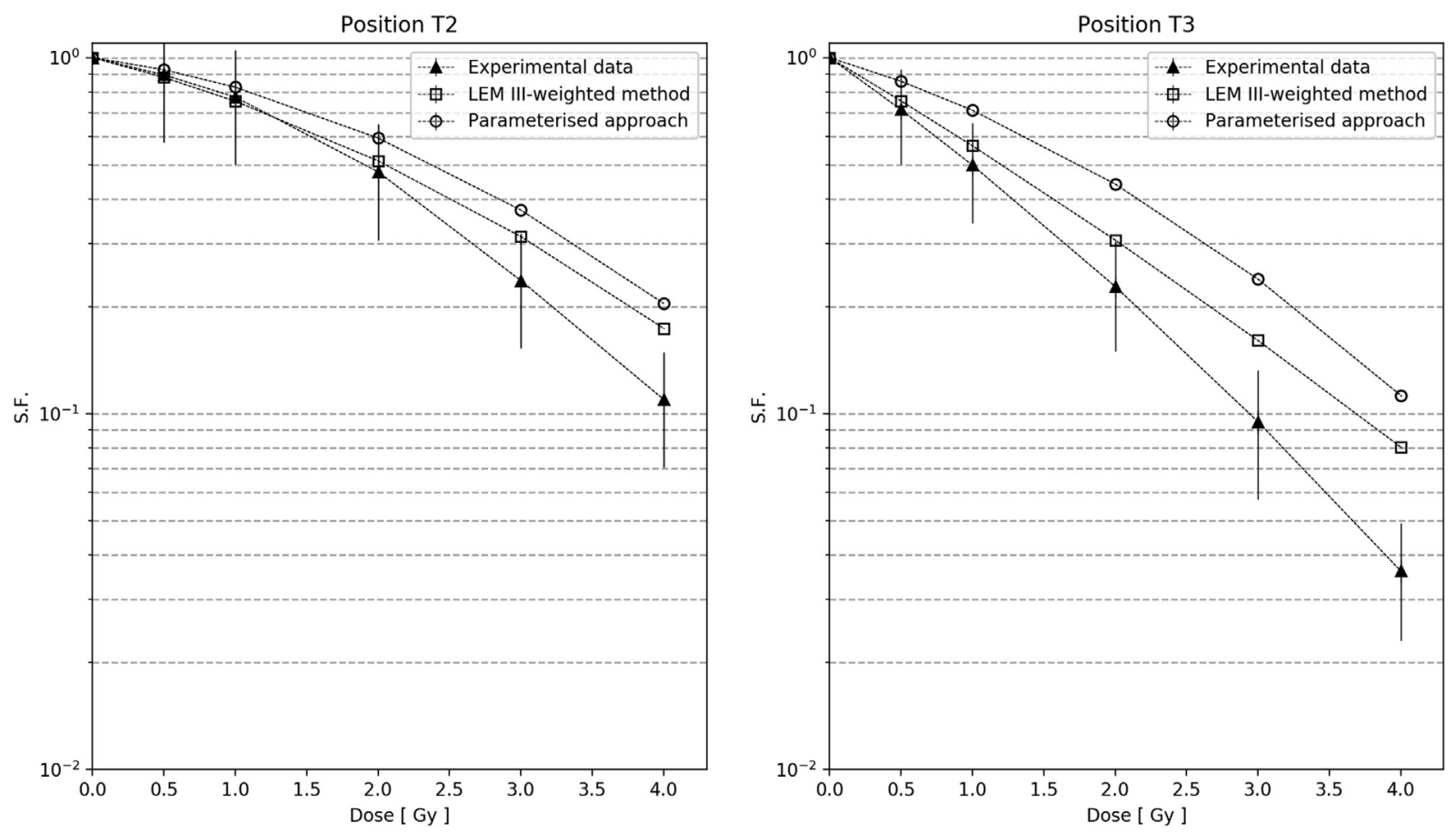

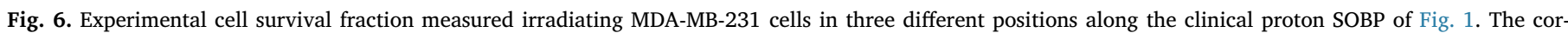
respondent curves calculated by using the Monte Carlo LEM III-weighted method (dashed lines) and the parameterised approach (dotted lines) are reported.

\subsection{Results for the U87 cell line}

The work from Chaudhary [3] reports the values of $\alpha_{D}$ and $\beta_{D}$ parameters, derived from the fit of the experimental data with the wellknown linear-quadratic expression (Eq. (3)) [33,34]. Individual uncertainties on $\alpha$ and $\beta$ are reported in the paper but not their correlation coefficient. U87 experimental survival curves, at the six positions along the $62 \mathrm{MeV}$ pristine Bragg peak, are reported in Fig. 3. The light-gray band represents the SF expectation (68\% coverage) based on the data by Chaudhary et al. [3]. The band was calculated by letting the parameters $\alpha$ and $\beta$ fluctuate according to the central values and the uncertainties reported in [3].

Survival curves calculated using the LEM (open squares) and the parameterized approach (open circles), are also reported. Survival curves for the same U87 cells, irradiated in six different positions along the clinical modulated SOBP, are reported in Fig. 4. The dose-response curves well reflect the increase in RBE with increasing proton LET, which rises from about $1 \mathrm{keV} / \mu \mathrm{m}$ to over $22 \mathrm{keV} / \mu \mathrm{m}$ from P1 to P6, with a progressively steeper slope as described by the $\alpha$ parameter. A significant increase in radiosensitivity with increasing depth along the 
Table 3

Results of the $\chi^{2}$ test for the comparison of the experimental survival curves of Fig. 4 against survival fraction calculated with the LEM III and parameterised approaches. The corresponding $\chi^{2}$ probabilities are calculated for four degrees of freedoms.

\begin{tabular}{ccccc}
\hline \multicolumn{5}{c}{ DU145 } \\
& \multicolumn{5}{c}{ LEM III weigthed method } & Parameterized approach \\
\hline Position & $\chi^{2}$ & p-value & $\chi^{2}$ & p-value \\
T1 & 4.14 & 0.38 & 6.06 & 0.19 \\
T2 & 4.23 & 0.37 & 5.95 & 0.20 \\
T3 & 5.14 & 0.27 & 5.32 & 0.25 \\
& & & & \\
& & MDA-MB-231 & & p-value \\
& & PEM III weigthed method & 0.05 \\
Position & $\chi^{2}$ & p-value & $\chi^{2}$ & $5.4 * 10^{-12}$ \\
T2 & 3.64 & 0.45 & 9.10 & \\
T3 & 15.85 & 0.03 & 58.70 & \\
& & & & \\
\hline
\end{tabular}

SOBP can be observed by the marked reduction of the shoulder (as described by the beta parameter) and an increasingly linear dependence of the surviving fraction upon dose (as described the alpha parameter), which becomes particularly evident in the curves obtained from cells irradiated in positions from P4 to P6. A similar trend can be observed for U87 cells irradiated along the SOBP, although in this case the increase in the dose-response curve initial slope is less marked compared to the monoenergetic beam. Based on the tabulated parameters for the experimental results (Table 1) obtained for the U87 cells, for example, the surviving fraction (SF) following $2 \mathrm{~Gy}$ decreased by almost twothirds between entrance and the peak position in the case of monoenergetic proton beam irradiation (i.e. from 0.61 to 0.16 ); the increase in radiosensitivity at the same dose when cells were irradiated in the SOBP configuration was about $50 \%$ since the SF almost halved (i.e.
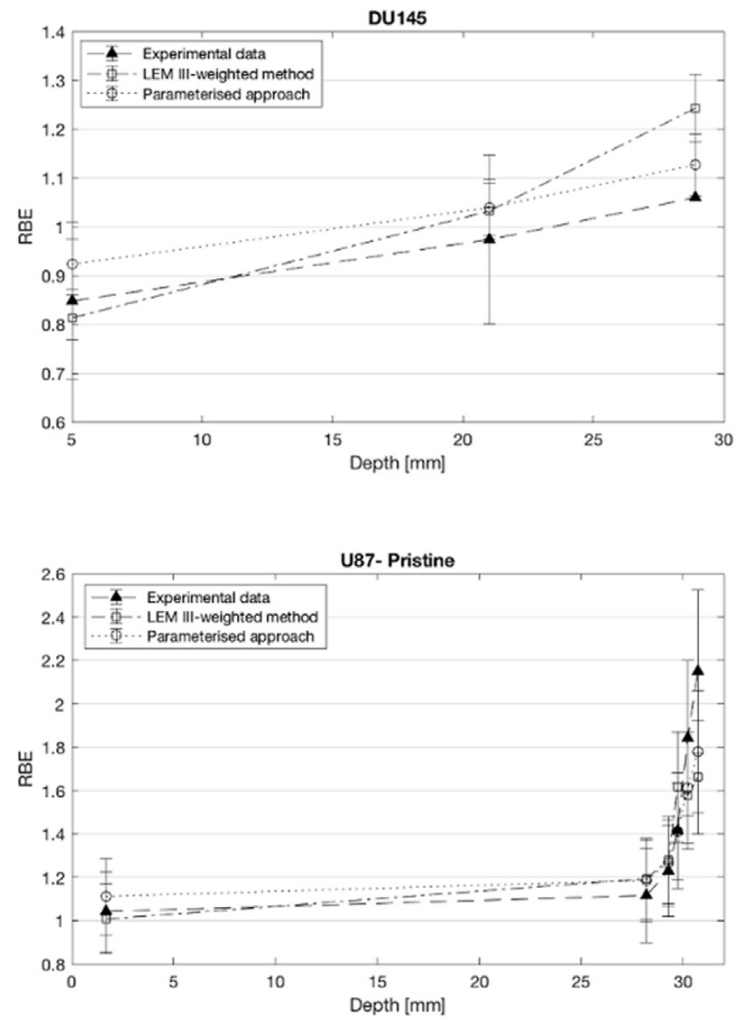

from 0.59 to 0.29 ) between entrance and the SOBP distal end. This is in line with the smaller LET difference between entrance and proton end range when passing from the pristine to SOBP configuration.

\subsection{DU145 and MDA-MB-231 cell line irradiations}

For the DU145 and MDA-MB-231 cells a direct comparison of the SFs at different doses was performed. In Table 2 reports the experimental $\alpha$ and $\beta$ parameters and the calculations based on the two approaches. Fig. 5 and Fig. 6 display the experimental survival fractions obtained for DU145 irradiated in the positions T1, T2 and T3 of the proton SOBP marked in Fig. 1, and for MDA-MB-231 cells irradiated at the positions $\mathrm{T} 2$ and $\mathrm{T} 3$ respectively. The same plots also report the results from Monte Carlo simulations, using the two calculation approaches. Error bars on experimental data were derived by taking into account the standard uncertainties affecting colony counting, cell dilution and plating efficiency. Specifically, the uncertainty $\sigma_{E}(S F)$ on the $\mathrm{SF}$ must take into account the normalization factor given by the socalled plating efficiency PE, which represents the colony-forming ability or SF of unirradiated cells:

$P E=\frac{X_{\text {colonies }}}{X_{\text {cells }}} \mid 0 G y$

$\sigma_{E}(S F)$ can be determined as follows:

$\sigma_{E}\left(S F_{D}\right)=\sqrt{\left(\frac{1}{P E} \sigma_{E_{\text {colonies }}} X_{\text {cells }}\right)^{2}+\left(-\frac{S F}{(P E)^{2}} \sigma_{E}(P E)\right)^{2}}$

Uncertainties on calculations (not visible in the Fig. 5 and Fig. 6) were obtained by combining the simulation statistical errors and the contributions coming from the propagation of the uncertainties of $\left\langle\alpha_{D}\right\rangle$ and $\left\langle\beta_{D}\right\rangle$ from the respective look-up tables.
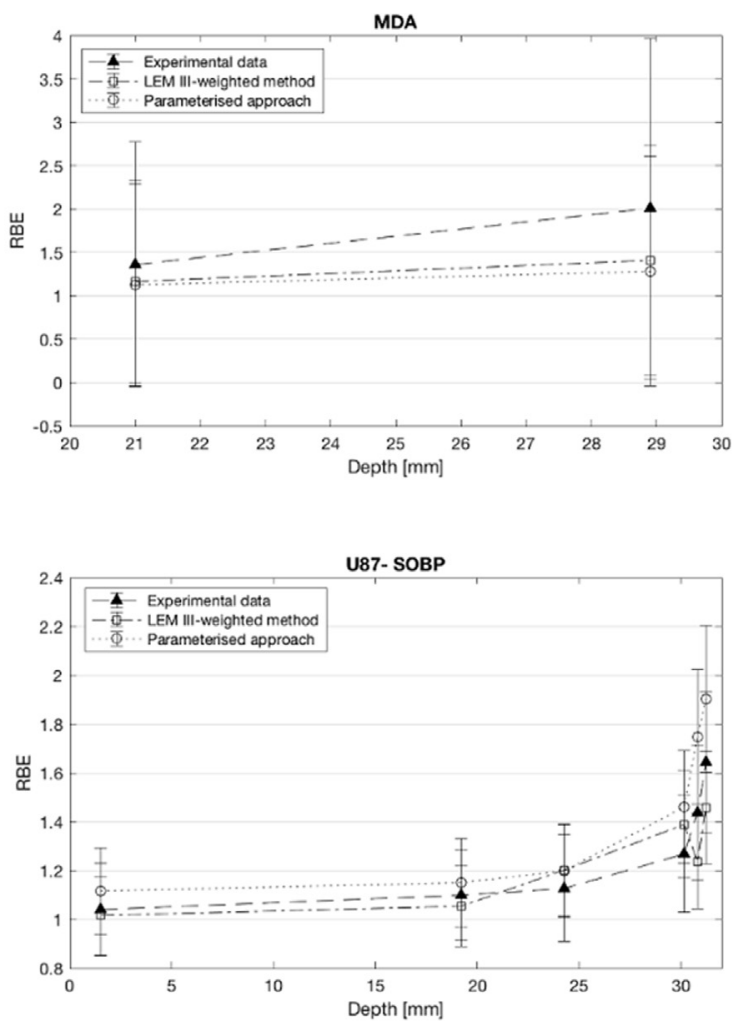

Fig. 7. Experimental RBE measured irradiating DU145, MDA-MB-231 and U87 cells in different positions along the Bragg curve. The values calculated by using the Monte Carlo LEM III-weighted method (triangle points) and the parameterised approach (dot points), are reported. 


\section{Discussion}

Experimental clonogenic curves for U87, DU154 and MDA-MB-231 cell lines, all irradiated along the same beam line were compared with the results obtained using two different computational methods: Monte Carlo LEM III-weighted method and a parameterised approach, for the estimation of the radioresponse following cellular exposure to protons. Cells were irradiated using a pristine $62 \mathrm{MeV}$ monochromatic Bragg peak and a clinical SOBP, at various depths in water corresponding to different LET values. The agreement between experimental and calculated curves is satisfactory for the three considered cell lines. As visible in Fig. 3, Fig. 4 and Table 3, a slighty discrepancy can be observed for the U87 cells at higher LET values, i.e. at greater depths of the particle beams. Such discrepancies may be connected with the uncertainty on flask window thickness and positioning, which becomes critical at the end of the particle range. It may also be connected to the wider particles energy spectra in that position. In the distal part of the Bragg curve, in fact, very small differences in depth (of the order of hundreds of $\mu \mathrm{m}$ ) can produce differences in the LET values up to $15 \%$.

For the DU145 and MDA-MB-231 cells, a $\chi^{2}$ test has been performed in order to quantify the agreement between experimental and simulated SFs. Results of the test are reported in Table 3: the $\chi^{2}$ values calculated over the four available data points $(0.5,1,2$ and $4 \mathrm{~Gy})$ and the corresponding p-values are shown for the three positions. For the DU145 cell lines, the resulted p-value is well above than 0.25 , showing that the models are able to reproduce the experimental data. The parametrized model under-estimates the SFs at high doses at the distal position T3, yielding a p-value around 0.05. The LEM model also predicts a lower SFs for the high doses at the distal position T3, but the difference is well within the statistical fluctuations.

The MDA-MB-231 cell lines result in accordance with experimental data for the position T2, corresponding to the MID-SOBP of the Bragg curve. The p-value calculated for the position T3, is lower than 0.05 as shown in Table 3. This disagreement is essentially due to the higher uncertainty of the cells positioning during the experimental campaign.

In general, the LEM III-weighted model shows a better agreement than the parametrized one; in all cases the most critical points were the distal ones, where positioning uncertainties are most significant.

The agreement between calculated and experimental data appears evident also for the RBE curves reported in Fig. 7. In all investigated cases the RBE prediction entirely falls within the experimental error bars.

In the Monte Carlo approach, a further source of uncertainties could be related to the contribution of the secondary particles. Although the LEM, in its original formulation, is already conceptually geared to be used considering the full spectra of primary and secondary particles, in the case of proton irradiation the contribution of secondary particles is still debated [35]. We remark however that one of the main goal of this paper is to present a general framework to include radiobiological evaluations in the Geant4 Monte Carlo simulations where is possible to evaluate the contribution of both primaries and secondary particles, based on a reliable knowledge of the relevant cross section data.

\section{Conclusion}

In this study we shown that a LEM-based and a parameterised approach can adequately reproduce experimentally derived survival curves from in vitro cancer cell irradiations performed in a clinical proton beam. Unlike similar computational codes, the open-source nature of the employed MC code arguably represents a valuable asset that is much needed in the expanding field of cancer hadrontherapy. An interesting future application field will be certainly related with similar studies performed with high-LET incident particles [36,37]. This preliminary work will be further extended with experimental data from other cancer cell lines irradiated at the CATANA beam line, in order to initiate a clinically relevant database and with the simulation and evaluation of cells damage at the DNA scale using computational approaches as reported in recent publications [38-40].

\section{Acknowledgment}

This work was supported by the MoVe IT (modeling and verification for ion beam treatment planning), NEPTUNE (nuclear process driven enhancement of proton therapy unraveled) and MC(Monte Carlo)-INFN projects funded by National Institute for Nuclear Physics (INFN). It was also supported by the ELI-Beamlines MEDical and multidisciplinary applications (ELIMED) project and by the ESA-AO7146 project.

\section{References}

[1] Website of the Particle Therapy CoOperative Group, web:https://www.ptcog.ch/.

[2] Paganetti H, Niemierko A, et al. Relative biological effectiveness (RBE) values for proton beam therapy. Int. J. Radiat. Oncol. Biol. Phys. 2002;53:407-21.

[3] Chaudhary P, Marshall TI, et al. Relative biological effectiveness variation along monoenergetic and modulated bragg peaks of a $62-\mathrm{MeV}$ therapeutic proton beam: a preclinical assessment. Int. J. Radiat. Oncol. Biol. Phys. 2014;90(1):27-35.

[4] Darby SC, Ewertz M, et al. Risk of ischemic heart disease in women after radiotherapy for breast cancer. New England J. Med. 2013;368(11):987-98.

[5] Bouchard $\mathrm{G}$, Therriault $\mathrm{H}$, et al. Induction of interleukin- 1 by mouse mammary tumor irradiation promotes triple negative breast cancer cells invasion and metastasis development. Int. J. Radiat. Biol. 2017;93(5):507-16.

[6] IAEA TRS 461 Relative Biological Effectiveness in Ion Beam Therapy, International Atomic Energy Agency, 2008.

[7] Scholz M, Kellerer AM, et al. Computation of cell survival in heavy ion beams for therapy. The model and its approximation. Radiat. Environ. Biophys. 1997;36:59-66.

[8] Hawkins RB. A microdosimetric-kinetic model of cell death from exposure to ionizing radiation of any LET, with experimental and clinical applications. Int. J. Radiat. Biol. 2017;69(6):739-55.

[9] Rossi S. The National center for Oncological hadrontherapy (CNAO): status and perspectives. Physica Med. 2015;31(4):333-51.

[10] Benedickt M, Fabich A. MedAustron - Austrian hadron therapy center. Nuclear Science Symposium Conference record 2008;5597-5599.

[11] Kramer M, et al. Treatment planning for heavy-ion radiotherapy: physical beam model and dose optimization. Phys. Med. Biol. 2000;45. 3299-3217.

[12] Kramer M, Scholz M. Treatment planning for heavy-ion radiotherapy: calculation and optimization of biologically effective dose. Phys. Med. Biol. 2000;45:3319-30.

[13] Carante MP, Ballarini F. Calculating variations in biological effectiveness for a $62 \mathrm{MeV}$ proton beam. Front. Oncol. 2016:6-76.

[14] Carlson DJ, Stewart RD, et al. Combined use of Monte Carlo DNA damage simulations and deterministic repair models to examine putative mechanisms of cell killing. Radiat. Res. 2008;169:447-59.

[15] Kase Y, Kanematsu N, et al. Biological dose calculation with Monte Carlo physics simulation for heavy-ion radiotherapy. Phys. Med. Biol. 2006;51:467-75.

[16] Mairani A, et al. The FLUKA Monte Carlo code coupled with the local effect model for biological calculations in carbon ion therapy. Phys. Med. Biol. 2010;55:4273-89.

[17] Elsasser T, Kramer M, Scholz M. Accuracy of the local effect model for the prediction of biologic effects of carbon ion beams in vitro and in vivo. Int. J. Radiat. Oncol. Biol. Phys. 2008;71:866-72.

[18] Elsasser T, Scholz M. Improvement of the local effect model (LEM) - implications of clustered DNA damage. Radiat. Prot. Dosimetry 2006;122(1-4):475-7.

[19] Elsasser T, Scholz M. Cluster effects within the local effect model. Radiat. Res. 2007;167:319-29.

[20] Kramer M, Scholz M. Rapid calculation of biological effects in ion radiotherapy. Phys. Med. Biol. 2006;51:1959-70.

[21] McNamara AL, Schuemann J, Paganetti H. A phenomenological relative biological effectiveness (RBE) model for proton therapy based on all published in vitro cell survival data. Phys. Med. Biol. 2015;60(21):8399-416.

[22] Cirrone GAP, Cuttone G, et al. Clinical and research activities at the CATANA facility of INFN-LNS: from the conventional hadrontherapy to the laser-driven approach. Front. Oncol. 2017;10.

[23] Cirrone GAP, Cuttone G, et al. Implementation of a new Monte Carlo GEANT4 simulation tool for the development of a proton therapy beam line and verification of the related dose distributions. IEEE Trans. Nucl. Sci. 2005;52:1756-8.

[24] Cirrone GAP, Cuttone G, et al. Hadrontherapy: a 4-based tool for proton/iontherapy studies. Progr. Nucl. Sci. Technol. 2011;2:207-12.

[25] Allison J, et al. Recent Developments in Geant4. Nucl. Instrum. Meth. A 2016;835:186-225.

[26] Scholz M, Kraft G. Track structure and the calculation of biological effects of heavy charged particles. Adv. Space Res. 1996;18:5-14.

[27] Scholz M, Kellerer A, et al. Computation of cell survival in heavy ion beams for therapy. Radiat. Environ. Biophys. 1997;36:59-66.

[28] Manganaro L, Russo G, et al. Survival: a simulation toolkit introducing a modular approach for radiobiological evaluations in ion beam therapy. Phys. Med. Biol. 2018;63(8). 08-01.

[29] Zaider M, Rossi H. The synergistic effects of different radiations. Radiait. Res. 
$1980 ; 83: 732-9$.

[30] Paganetti H. Relative biological effectiveness (RBE) values for proton beam therapy. Variations as a function of biological endpoint, dose, and linear energy transfer. Phys. Med. Biol. 2014;59.

[31] Cortes-Giraldo M, Carabe A. A critical study of different Monte Carlo scoring methods of dose average linear-energy-transfer maps calculated in voxelized geometries irradiated with clinical proton beams. Phys. Med. Biol. 2015;60:2645-69.

[32] International Atomic Energy Agency, TRS-398, 12, 2006.

[33] Chadwick KH, Leenhouts HP. A molecular theory of cell survival. Phys. Med. Biol. 1973;13:78-87.

[34] Fertil B, Reydellet I, Deschavanne PJ. A benchmark of cell survival models using survival curves for human cells after completion of repair of potentially lethal damage. Radiat. Res. 1994;138:61-9.
[35] Tommasino F, Durante M. Proton radiobiology. Cancers 2015.

[36] Taleei R, Guan F, et al. Monte Carlo simulations of He ion physical characteristics in a water phantom and evaluation of radiobiological effectiveness. Med. Phys. 2016;43(2):761-76

[37] Mayu I, Yukari Y, et al. Carbon-ion beams effectively induce growth inhibition and apoptosis in human neural stem cells compared with glioblastoma A172 cells. J. Radiat. Res. 2015;56(5):856-61.

[38] Lampe N, Karamitros M, et al. Mechanistic DNA damage simulations in Geant4DNA Part 2: Electron and proton damage in a bacterial cell. Physica Med. 2018;12.

[39] Tello JJ, Incerti S, et al. Numerical insight into the Dual Radiation Action Theory. Physica Med. 2017;43:120-6.

[40] McNamara A, Geng C, et al. Validation of the radiobiology toolkit TOPAS-nBio in simple DNA geometries. Physica Med. 2017;33:207-15. 